\title{
Eficiência de utilização de nutrientes por cultivares de cafeeiro
}

\author{
Nutrients use efficiency by coffee cultivars
}

\section{José Francisco Teixeira do Amaral ${ }^{\mathrm{I}}$ Herminia Emilia Prieto Martinez ${ }^{\mathrm{II}}$ Bruno Galvêas Laviola ${ }^{\mathrm{III}}$ Elpidio Inácio Fernandes Filho ${ }^{\mathrm{IV}}$ Cosme Damião Cruz ${ }^{\mathrm{V}}$}

RESUMO

Elucidar as diferenças nas exigências nutricionais entre as cultivares é uma forma de obter maior produtividade e otimizar o uso de fertilizantes. Este trabalho teve por objetivo avaliar a eficiência na produção de raiz e parte aérea por unidade absorvida de $\mathrm{N}, \mathrm{P}, \mathrm{K}, \mathrm{Ca}, \mathrm{Mg}, \mathrm{S}, \mathrm{B}, \mathrm{Cu}$ e $\mathrm{Zn}$ em quatro cultivares de cafeeiro arábico ('Acaiá IAC-474-19', 'Icatu Amarelo IAC-3282', 'Rubi MG-1192'e 'Catuaí Vermelho IAC99'). Para tanto, foi conduzido um experimento em condições de campo, no Campus da Universidade Federal de Viçosa, durante dois anos, em delineamento experimental de blocos casualizados, em arranjo fatorial $4 \times 3$, constituído de quatro cultivares e três níveis de adubação (baixo, normal e alto), com quatro repetições. As plantas que constituíram o nível normal receberam adubação baseada na marcha de acúmulo de nutrientes em café arábica. Nos níveis de adubação baixo e alto, as plantas receberam, respectivamente, 0,4 e 1,4 vezes a recomendação de adubação feita para o nível normal. A eficiência de utilização de nutrientes para produção de raízes foi diferenciada entre as cultivares quando houve restrição na quantidade de adubos fornecidos (nível baixo), não havendo diferenças entre elas quando se empregou dose normal e alta de fertilizantes. A eficiência na produção de raízes por unidade de $\mathrm{N}, \mathrm{P}, \mathrm{K}, \mathrm{Ca}, \mathrm{Mg}$ e $\mathrm{S}$ absorvidos foi maior na cultivar 'Acaiá IAC-474-19' e menor na 'Rubi MG-1192'. Conclui-se que a eficiência de utilização de nutrientes para produção de raízes e uso de nutrientes pela parte aérea de cafeeiros foi diferenciada entre cultivares.

Palavras-chave: Coffea arabica L., nutrição mineral, eficiência nutricional, fertilizantes.

\begin{abstract}
To elucidate the differences in the nutritional requirements among the cultivated varieties of plant species is a form of obtaining higher productivity and to optimize the fertilizer use. This work had as objective to evaluate the root and shoot production efficiency of four arabic coffee cultivars ('Acaiá IAC 474-19', 'Icatú Amarelo IAC-3282', 'Rubi MG1192 ' and 'Catuaí Vermelho IAC 99') per unit of $N, P, K, C a$, $\mathrm{Mg}, \mathrm{S}, \mathrm{B}, \mathrm{Cu}$ and $\mathrm{Zn}$ absorbed. For this purpose an experiment was carried out in field conditions at the Universidade Federal de Viçosa. The treatments were settled in a $4 \times 3$ factorial arrangement (four cultivars and three fertilization levels; low, normal and high) in randomized blocks with four replications. The plants of the normal fertilization level received fertilization based on previously determined coffee plant recruitment. The plants of the levels low and high received, respectively, 0.4 and 1.4 times the normal fertilization doses. The efficiency of production of roots was differentiated among them cultivate when there was restriction in the amount of supplied fertilizers (low level), did not have differences among them when normal and high dose of fertilizers was used. When cultivate in the low fertilization level 'Acaiá IAC 474-19' presented highest efficiency to the production of roots per unit of $\mathrm{N}, \mathrm{P}, \mathrm{K}, \mathrm{Ca} \mathrm{Mg}$ and $\mathrm{S}$ absorbed, while in the some condition 'Rubi MG-1192' presented the smallest. It was concluded that efficiency of utilization of nutrients for root and shoot productions were differentiated among cultivars.
\end{abstract}

Key words: Coffea arabica L., mineral nutrition, nutritional efficiency.

'Centro de Ciências Agrárias, Universidade Federal do Espírito Santo (UFES), Alto Universitário, 29500-000, Alegre, ES, Brasil. E-mail: jfamaral@cca.ufes.br. Autor para correspondência.

"Departamento de Fitotecnia, Universidade Federal de Viçosa (UFV), Viçosa, MG, Brasil.

"I'Embrapa Agroenergia, Parque Estação Biológica (PqEB) Brasília, DF, Brasil.

${ }^{\mathrm{IV}}$ Departamento de Solos, UFV, Viçosa, MG, Brasil.

${ }^{\vee}$ Departamento de Biologia Geral, UFV, Viçosa, MG, Brasil. 


\section{INTRODUÇÃO}

O termo 'eficiência nutricional' é utilizado para caracterizar plantas em sua capacidade de absorver e utilizar nutrientes, estando relacionado à eficiência de absorção, translocação e utilização de nutrientes. A eficiência de absorção está relacionada à taxa de absorção de nutrientes por unidade de comprimento ou de massa de raiz, e pode ser avaliada em estudos de cinética de absorção de nutrientes (BALIGAR \& FAGERIA, 1998). A maior ou menor produção de frutos por unidade de nutrientes na planta pode ser explicada por diferenças na eficiência de aquisição, de translocação e/ou de utilização dos nutrientes. A aquisição de nutrientes depende da eficiência dos mecanismos de absorção e do volume de solo explorado pelas raízes, e pode ser avaliada pelas eficiências de absorção e de produção de raízes. Por sua vez, a eficiência de utilização de nutrientes depende do seu transporte para a parte aérea e de sua exigência metabólica (MARTINEZ et al., 1993), sendo obtida pelo quociente entre a biomassa total da planta e a concentração do nutriente (SIDDIQI \& GLASS, 1981). Tradicionalmente, a eficiência de utilização de nutriente tem sido definida como a razão entre a biomassa e a quantidade total de nutriente na biomassa (TOMAZ \& AMARAL, 2008).

O café é o principal produto comercializado em todo o mundo, depois do petróleo, com vendas globais alcançando cerca de noventa bilhões de dólares, sendo o Brasil o maior produtor mundial. Cerca de $70 \%$ da produção mundial é proveniente de pequenas propriedades rurais, envolvendo a mão-deobra familiar (DaMATTA et al., 2007).

Pouco se conhece sobre a eficiência nutricional de cafeeiros, embora muitas informações com relação à nutrição mineral sejam encontradas na literatura, como a absorção, o transporte e a redistribuição de nutrientes, que apresentam controle genético, existindo a possibilidade de melhorar e/ou selecionar cultivares mais eficientes quanto ao uso de nutrientes (GABELMAN \& GERLOFF, 1983).

A investigação da eficiência nutricional de $\mathrm{Ca}, \mathrm{Mg}$ e $\mathrm{S}$ no cafeeiro se reveste de grande importância, tendo em vista que os solos de fronteiras agrícolas para a cultura do café, em sua maioria, apresentam acidez elevada, baixa capacidade de retenção de cátions (CTC) e baixos teores de bases trocáveis, como $\mathrm{Ca}$ e $\mathrm{Mg}$ e frequentemente de $\mathrm{S}$ (TOMAZ et al., 2003). O acúmulo desses três nutrientes em frutos de café foi estudado por LAVIOLA et al. (2007), que verificaram maiores acúmulos relativos de $\mathrm{Ca}, \mathrm{Mg}$ e S no estádio de granação-maturação.
A cultivar 'Catuaí', comparada à 'Mundo Novo', é menos exigente em $\mathrm{Ne}$ e, mais exigente em $\mathrm{K}$, $\mathrm{Ca}, \mathrm{B}, \mathrm{Fe}, \mathrm{Mn}$ e Zn, e praticamente apresentam a mesma exigência em $\mathrm{Mg}, \mathrm{S}$ e Cu (MALAVOLTA, 1993). Por sua vez, a 'Mundo Novo', quando comparada à 'Rubi MG-1192', é menos eficiente na utilização de S (SOUZA, 1999). PEREIRA (1999) também discriminou linhagens de cafeeiros arábicos com base na eficiência de utilização de N e K.

É importante ressaltar que o conhecimento dos teores de nutrientes em vários órgãos do vegetal permite inferir sobre as exigências metabólicas desenvolvidas em cada compartimento, fornecendo base para o entendimento dessas variações e suas implicações nas respostas dos vegetais.

Assim, o objetivo deste trabalho foi avaliar a eficiência de utilização de nutrientes para produção de raízes e parte aérea por quatro cultivares de café arábico cultivadas na região da Zona da Mata de Minas Gerais, visando discriminar cultivares eficientes no uso de nutrientes.

\section{MATERIAL E MÉTODOS}

O experimento foi conduzido em Área Experimental da Agronomia, localizada no Campus Universitário, pertencente à Universidade Federal de Viçosa, num latossolo vermelho-amarelo distrófico, sendo instalado no dia 20/10/1998, com duração de dois anos. Foram utilizadas quatro cultivares de café arábica: 'Acaiá IAC-474-19', 'Icatu Amarelo IAC-3282', 'Rubi MG-1192'e 'Catuaí Vermelho IAC-99'.

O experimento constituiu-se de três níveis de adubação e correção da acidez do solo: baixo, normal (recomendado para a cultura) e alto. O delineamento experimental empregado foi em blocos completos casualizados e os tratamentos foram distribuídos em arranjo fatorial $4 \times 3$ (quatro cultivares de café e três níveis de adubação), com quatro repetições. Considerou-se como área útil da parcela as nove plantas dispostas no centro das três fileiras centrais da parcela. As análises estatísticas foram realizadas pelo programa GENES (CRUZ, 2001) e os dados foram submetidos à análise de variância e ao teste de Tukey ao nível de $10 \%$ de probabilidade.

As plantas que constituíam o nível normal receberam $\mathrm{N}, \mathrm{P}$ e K com base na marcha de acúmulo, considerando-se a média de nutrientes acumulados para as cultivares 'Mundo Novo'e 'Catuaí' (MALAVOLTA, 1993). O Ca e Mg foram fornecidos via calcário dolomítico com base em análises de solo, considerandose $60 \%$ de saturação em bases como ideal para o cafeeiro (GUIMARÃES et al., 1999). O enxofre foi 
fornecido como elemento acompanhante de fertilizantes nitrogenados e fosfatados.

A aplicação de corretivos e fertilizantes para o nível normal constou do seguinte: a) Plantio: $20 \mathrm{~g}$ $\operatorname{cova}^{-1}$ de calcário dolomítico e $48 \mathrm{~g} \mathrm{cova}^{-1}$ de $\mathrm{P}_{2} \mathrm{O}_{5}$; b) Pós-plantio: $8 \mathrm{~g} \mathrm{cova}^{-1}$ de $\mathrm{N}$ em três aplicações e $20 \mathrm{~g}$ $\operatorname{cova}^{-1}$ de $\mathrm{K}_{2} \mathrm{O}$ em duas aplicações, em intervalos de 30 dias; c) Correção da acidez do solo: foi realizada anualmente mediante aplicação manual de calcário dolomítico na faixa de plantio, considerando-se uma profundidade de incorporação de $5 \mathrm{~cm}$; d) Adubação de primeiro, segundo e terceiro ano: foram realizadas via fertirrigação, considerando-se uma eficiência de recuperação de $90 \%$ para o N, $80 \%$ para o Ke $70 \%$ para o P. Os nutrientes $\mathrm{N}, \mathrm{P}$ e $\mathrm{K}$ foram aplicados ao solo localizadamente pela utilização de fertirrigação por gotejamento, com o suporte do software SISDA $_{\text {café }}$ (MANTOVANI \& COSTA, 1998). O N foi fornecido pela aplicação alternada de sulfato de amônio, uréia e nitrato de amônio; o P pela aplicação de MAP (fosfato monoamônico); o K foi adicionado por meio do cloreto de potássio. Um quarto da exigência nutricional anual calculada foi aplicado no período de abril a agosto, e os 3/4 restantes, de setembro a março; e) Micronutrientes: $\mathrm{Zn}$, B e Cu foram supridos por meio de uma aplicação foliar anual, utilizando-se sulfato de zinco, ácido bórico e oxicloreto de cobre, na concentração de $4 \mathrm{~g} \mathrm{~L}^{-1}$ para o nível normal. Nos níveis baixo e alto, as plantas receberam, respectivamente, 0,4 e 1,4 vezes a recomendação feita para o nível normal.

As avaliações experimentais foram iniciadas no mês de maio de 2001, coincidindo com a colheita dos frutos. Para o estudo das eficiências nutricionais, foi utilizada uma planta em cada unidade experimental, cuja parte aérea foi fracionada em caule, ramos e folhas. Para estimar o comprimento e a matéria seca de raízes, foram retiradas 12 amostras de uma planta em cada parcela, obtidas a distâncias de 15, 30 e 45cm do tronco, no sentido dos quatro pontos cardeais, a $40 \mathrm{~cm}$ de profundidade, utilizando-se um trado cilíndrico de $4,5 \mathrm{~cm}$ de diâmetro. As amostras obtidas foram lavadas para separar as raízes do solo, identificadas e dispostas em lâmina de vidro para processamento das imagens em um microcomputador, ligado a um scanner HP Scanjet 4C. As imagens obtidas foram processadas pelo software Quantroot elaborado pelo Departamento de Solos da Universidade Federal de Viçosa, especialmente desenvolvido para determinação do comprimento de raízes, que, após determinado, os resultados foram extrapolados para o volume de solo ocupado pela planta $\left(1,25664 \mathrm{~m}^{3}\right)$, considerando-se esse volume igual ao de um cilindro com raio de $1 \mathrm{~m}$ (projeção da copa) e profundidade de 0,40m. Após o processamento das imagens, as amostras foram secas em estufa de circulação forçada de ar a $70^{\circ} \mathrm{C}$, até peso constante, para determinação da matéria seca das raízes. Esse peso foi também extrapolado para o volume de solo ocupado pela planta. Foram, então, separadas amostras desse material para determinação dos teores e conteúdos dos nutrientes.

As amostras da parte aérea foram identificadas, lavadas com água deionizada, acondicionadas em sacos de papel e secas em estufa com circulação forçada de ar, a $70^{\circ} \mathrm{C}$, até peso constante, conforme descrito por JONES JUNIOR et al. (1991). A seguir, amostras dos materiais vegetais secos foram moídas em moinho tipo Wiley, de aço inoxidável, passadas em peneira de malha de $0,841 \mathrm{~mm}$, e acondicionadas em sacos de papel, para posterior determinação dos teores e conteúdos dos elementos minerais.

Os teores de macronutrientes e de $\mathrm{Cu}, \mathrm{Zn}$ e $\mathrm{B}$ foram determinados em todas as partes vegetativas da planta, a saber: raízes, caule, folhas e ramos. Os índices de eficiência nutricional foram obtidos a partir dos pesos da matéria seca e os teores de nutrientes de cada parte da planta, conforme as relações apresentadas a seguir (AMARAL, 2002):

Eficiência de utilização de nutrientes para produção de raízes (EPR)

$\mathrm{EPR}=\frac{(\text { Matéria seca de raiz) })^{2}}{\text { Conteúdo de nutriente na planta toda }}, \mathrm{em} \mathrm{g}^{2} \mathrm{mg}^{-1} \mathrm{e} \mathrm{g}^{2} \mathrm{mg}^{-1}$

para macro e micronutrientes, respectivamente.

Eficiência de utilização de nutrientes para produção de parte aérea (EUPA)

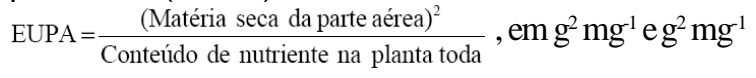

para macro e micronutrientes, respectivamente.

Eficiência de utilização de nutrientes para produção de folhas (EUF)

$\mathrm{EUF}=\frac{(\text { Matéria seca de folha })^{2}}{\text { Conteúdo de nutriente na parte aérea }}, \mathrm{em} \mathrm{g}^{2} \mathrm{mg}^{-1} \mathrm{e} \mathrm{g}^{2} \mathrm{mg}^{-1}$

para macro e micronutrientes, respectivamente.

Eficiência de utilização de nutrientes para produção de caule (EUC)

$\mathrm{EUC}=\frac{(\text { Matéria seca do caule })^{2}}{\text { Conteúdo de nutriente na parte aérea }}, \mathrm{em} \mathrm{g}^{2} \mathrm{mg}^{-1} \mathrm{e} \mathrm{g}^{2} \mathrm{mg}^{-1}$

para macro e micronutrientes, respectivamente.

Eficiência de utilização de nutrientes para produção de ramos (EUR)

$\mathrm{EUR}=\frac{(\text { Matéria seca de ramos) })^{2}}{\text { Conteúdo de nutriente na parte aérea }}, \mathrm{em} \mathrm{g}^{2} \mathrm{mg}^{-1} \mathrm{e} \mathrm{g}^{2} \mathrm{mg}^{-1}$

para macro e micronutrientes, respectivamente.

\section{RESULTADOS E DISCUSSÃO}

A eficiência de utilização de nutrientes para produção de raízes foi diferenciada entre as cultivares 
quando houve restrição na quantidade de adubos fornecidos (nível baixo), não havendo diferenças entre elas quando se empregou dose normal e alta de fertilizantes. Quando se utilizou 0,4 vez a dose estabelecida para o nível normal, a cultivar 'Acaiá IAC474-19' apresentou maior produção de raízes por unidade de $\mathrm{N}, \mathrm{P}, \mathrm{K}, \mathrm{Ca}, \mathrm{Mg}$ e $\mathrm{S}$ absorvidos, enquanto que a cultivar 'Rubi MG-1192' exibiu menor eficiência de utilização de nutrientes para produção de raízes, ficando a 'Icatu Amarelo IAC-3282'e a 'Catuaí Vermelho IAC-99' em posições intermediárias, sem diferir estatisticamente das demais cultivares (Tabela 1). Entretanto, maior eficiência de utilização de nutrientes para produção de raízes da cultivar 'Acaiá IAC-474-19' no nível baixo de adubação para todos os nutrientes (Tabela 1) não refletiu em maior produtividade desta cultivar neste ambiente, a qual apresentou menor eficiência agronômica (AMARAL, 2002).

Grande parte das pesquisas envolvendo a eficiência nutricional evidencia a eficiência de utilização de fósforo. Na presente pesquisa, o aumento da disponibilidade de nutrientes não permitiu detectar diferenças entre as cultivares com relação à eficiência de utilização de nutrientes para produção de raízes (Tabela 1), provavelmente devido ao concomitante aumento de massa de matéria seca de raiz. A produção de matéria seca de raízes em genótipos de arroz elevouse com o aumento da disponibilidade de P, embora o comprimento do sistema radicular não tenha sido alterado (FAGERIA et al., 1988). O comprimento do sistema radicular do milho também não foi afetado pelo suprimento de $\mathrm{P}$, ocorrendo, contudo, redução na área superficial e aumento no diâmetro das raízes com maior suprimento (SCHENK \& BARBER, 1979). Não obstante, FURLANI et al. (1983), estudando eficiência de absorção e de utilização de $\mathrm{P}$ em arroz, verificaram reduções no comprimento radicular e aumento no número de raízes principais e secundárias com o aumento da dose fornecida. Redução no comprimento radicular e na densidade de pêlos radiculares, com consequente redução na área superficial de raízes, devido ao aumento no suprimento de P, também foi notado por FÖHSE \& JUNGK (1983). Nesse aspecto, observa-se que a produção de matéria seca é importante na absorção de $\mathrm{P}$ quando associada à grande área superficial de raízes, uma vez que sua disponibilidade é afetada por intensa adsorção pelas partículas do solo (DOBERMANN et al., 1998).

A 'Acaiá IAC-474-19' apresentou maior eficiência de utilização de nutrientes para produção de caule para todos os nutrientes nos três níveis de adubação. Entretanto, a 'Icatu Amarelo IAC-3282', que foi a mais produtiva no nível baixo de adubação
(AMARAL, 2002), apresentou a segunda maior eficiência de utilização de nutrientes para produção de caule em todos os níveis, exceto para $\mathrm{P}$ no nível alto (Tabela 2). Ao contrário, cultivares de porte baixo como 'Rubi MG-1192' e 'Catuaí Vermelho IAC-99' apresentaram menor eficiência de utilização de nutrientes para produção de caule (Tabela 2) e foram mais produtivas, principalmente nos níveis normal e alto de adubação (AMARAL, 2002), as quais alocam menores quantidades de nutrientes no caule, investindo mais fotoassimilados no crescimento e desenvolvimento de outros órgãos, tais como folhas, de importância na produção de compostos orgânicos necessários aos processos de florescimento e frutificação.

A eficiência de utilização de nutrientes para produção de ramos (Tabela 3) evidenciou diferenças entre cultivares somente para o N, S e Zn no nível baixo de adubação, com destaque para a 'Icatu Amarelo IAC-3282', que apresentou maior eficiência. A 'Catuaí Vermelho IAC-99' foi a menos eficiente, enquanto que as demais cultivares se posicionaram intermediariamente quanto a esses nutrientes.

Poder-se-ia inferir que a maior ou menor produção de matéria seca de ramos fosse acompanhada, respectivamente, de maior ou menor produção de matéria seca de folhas. Para a 'Catuaí Vermelho IAC99' isso se verificou, visto que apresentou menor eficiência de utilização de nutrientes para produção de ramos e de folhas, no menor nível de adubação. No entanto, isso não se aplica às demais cultivares, principalmente para a 'Icatu Amarelo IAC-3282', mais eficiente na utilização de nutrientes para produção de ramos e também a mais produtiva no nível baixo de adubação (AMARAL, 2002), apresentando, no entanto, eficiência de utilização de nutrientes intermediária para produção de folhas (Tabelas 3 e 4 , respectivamente).

Nesse aspecto, é prudente questionar se deve ou não atrelar a eficiência de utilização de nutrientes para produção de folhas ao aumento da produtividade vegetal. É importante ressaltar a importância da área foliar por ser um parâmetro indicativo de produtividade, pois o processo fotossintético depende da interceptação da energia luminosa e sua conversão em energia química (FAVARIN et al., 2002). Torna-se relevante ressaltar que diferenças genotípicas na eficiência nutricional estão relacionadas à absorção, ao transporte e à redistribuição de nutrientes (GABELMAN \& GERLOFF, 1983), sendo esta uma maneira de diminuir perda de nutrientes pelas plantas, permitindo a manutenção das atividades metabólicas, principalmente em períodos sujeitos ao estresse nutricional. 
Tabela 1 - Médias de eficiência de utilização de nutrientes para produção de raízes, em $\mathrm{g}^{2} \mathrm{mg}^{-1} \mathrm{e} \mathrm{g}^{2} \mu \mathrm{g}^{-1}$ para macro e micronutrientes, respectivamente, de quatro cultivares de café arábica, tratadas com três níveis de adubação.

\begin{tabular}{|c|c|c|c|c|c|c|}
\hline \multirow{2}{*}{ Nutriente } & \multirow{2}{*}{ Cultivar } & \multicolumn{3}{|c|}{----------------Nível de Adubação---------------- } & \multirow{2}{*}{ Média } & \multirow{2}{*}{$\mathrm{CV}(\%)$} \\
\hline & & Baixo & Normal & Alto & & \\
\hline \multirow[t]{5}{*}{$\mathrm{N}$} & ‘Acaiá IAC-474-19’ & $5,5689^{\mathrm{A}}$ & $1,9692^{\mathrm{A}}$ & $1,4953^{\mathrm{A}}$ & $3,0111^{\mathrm{A}}$ & \multirow{5}{*}{104,32} \\
\hline & 'Icatu Amarelo IAC-3282' & $2,9247^{\mathrm{AB}}$ & $2,3823^{\mathrm{A}}$ & $1,4708^{\mathrm{A}}$ & $2,2593^{\mathrm{A}}$ & \\
\hline & 'Rubi MG-1192' & $1,0344^{\mathrm{B}}$ & $3,7423^{\mathrm{A}}$ & $1,1696^{\mathrm{A}}$ & $1,9821^{\mathrm{A}}$ & \\
\hline & 'Catuaí Vermelho IAC-99' & $1,7670^{\mathrm{AB}}$ & $2,3932^{\mathrm{A}}$ & $1,3527^{\mathrm{A}}$ & $1,8376^{\mathrm{A}}$ & \\
\hline & Média & $2,8237^{\text {a }}$ & $2,6218^{\mathrm{a}}$ & $1,3721^{\mathrm{a}}$ & & \\
\hline \multirow[t]{5}{*}{$\mathrm{P}$} & ‘Acaiá IAC-474-19’ & $66,5351^{\mathrm{A}}$ & $33,7221^{\mathrm{A}}$ & $25,3579^{\mathrm{A}}$ & $41,8717^{\mathrm{A}}$ & \multirow{5}{*}{90,34} \\
\hline & 'Icatu Amarelo IAC-3282' & $39,0095^{\mathrm{AB}}$ & $40,7412^{\mathrm{A}}$ & $27,5845^{\mathrm{A}}$ & $35,7784^{\mathrm{A}}$ & \\
\hline & 'Rubi MG-1192' & $11,5718^{\mathrm{B}}$ & $61,6622^{\mathrm{A}}$ & $23,4168^{\mathrm{A}}$ & $32,2170^{\mathrm{A}}$ & \\
\hline & ‘Catuaí Vermelho IAC-99’ & $19,8130^{\mathrm{AB}}$ & $39,7501^{\mathrm{A}}$ & $24,6135^{\mathrm{A}}$ & $28,0589^{\mathrm{A}}$ & \\
\hline & Média & $34,2324^{a}$ & $43,9689^{\mathrm{a}}$ & $25,2432^{\mathrm{a}}$ & & \\
\hline \multirow[t]{5}{*}{ S } & ‘Acaiá IAC-474-19’ & $59,7943^{\mathrm{A}}$ & $25,9727^{\mathrm{A}}$ & $17,9812^{\mathrm{A}}$ & $34,5827^{\mathrm{A}}$ & \multirow{5}{*}{90,70} \\
\hline & 'Icatu Amarelo IAC-3282' & $34,8202^{\mathrm{AB}}$ & $30,9986^{\mathrm{A}}$ & $19,2692^{\mathrm{A}}$ & $28,3627^{\mathrm{A}}$ & \\
\hline & 'Rubi MG-1192' & $11,5429^{\mathrm{B}}$ & $47,2775^{\mathrm{A}}$ & $14,3471^{\mathrm{A}}$ & $24,3892^{A}$ & \\
\hline & 'Catuaí Vermelho IAC-99' & $20,3117^{\mathrm{AB}}$ & $30,4323^{\mathrm{A}}$ & $17,9186^{\mathrm{A}}$ & $22,8875^{\mathrm{A}}$ & \\
\hline & Média & $31,6173^{\mathrm{a}}$ & $33,6703^{\mathrm{a}}$ & $17,3790^{\mathrm{a}}$ & & \\
\hline \multirow[t]{5}{*}{ K } & ‘Acaiá IAC-474-19’ & $14,2950^{\mathrm{A}}$ & $4,9884^{\mathrm{A}}$ & $3,6523^{\mathrm{A}}$ & $7,6452^{\mathrm{A}}$ & \multirow{5}{*}{115,31} \\
\hline & 'Icatu Amarelo IAC-3282' & $6,6394^{\mathrm{AB}}$ & $5,5194^{\mathrm{A}}$ & $3,4485^{\mathrm{A}}$ & $5,2024^{\mathrm{A}}$ & \\
\hline & 'Rubi MG-1192' & $2,2802^{\text {B }}$ & $9,4000^{\mathrm{A}}$ & $2,8707^{\mathrm{A}}$ & $4,8503^{\mathrm{A}}$ & \\
\hline & ‘Catuaí Vermelho IAC-99’ & $3,8270^{\mathrm{AB}}$ & $5,7304^{\mathrm{A}}$ & $3,0840^{\mathrm{A}}$ & $4,2138^{\mathrm{A}}$ & \\
\hline & Média & $6,7604^{a}$ & $6,4096^{\mathrm{a}}$ & $3,2639^{\mathrm{a}}$ & & \\
\hline \multirow[t]{5}{*}{$\mathrm{Ca}$} & ‘Acaiá IAC-474-19’' & $14,5743^{\mathrm{A}}$ & $6,3488^{\mathrm{A}}$ & $5,2377^{\mathrm{A}}$ & $8,7203^{\mathrm{A}}$ & \multirow{5}{*}{102,53} \\
\hline & 'Icatu Amarelo IAC-3282' & $7,1265^{\mathrm{AB}}$ & $6,8595^{\mathrm{A}}$ & $5,4009^{\mathrm{A}}$ & $6,4623^{A}$ & \\
\hline & 'Rubi MG-1192' & $2,1988^{\mathrm{B}}$ & $11,5647^{\mathrm{A}}$ & $3,9906^{\mathrm{A}}$ & $5,9180^{\mathrm{A}}$ & \\
\hline & ‘Catuaí Vermelho IAC-99’ & $4,4245^{\mathrm{AB}}$ & $6,1846^{\mathrm{A}}$ & $4,7883^{\mathrm{A}}$ & $5,1325^{\mathrm{A}}$ & \\
\hline & Média & $7,0810^{\mathrm{a}}$ & $7,7394^{\mathrm{a}}$ & $4,8544^{\mathrm{a}}$ & & \\
\hline \multirow[t]{5}{*}{$\mathrm{Mg}$} & 'Acaiá IAC-474-19’ & $33,8401^{\mathrm{A}}$ & $14,6579^{\mathrm{A}}$ & $11,3493^{\mathrm{A}}$ & $19,9491^{\mathrm{A}}$ & \multirow{5}{*}{90,96} \\
\hline & 'Icatu Amarelo IAC-3282' & $17,5963^{\mathrm{AB}}$ & $16,9362^{\mathrm{A}}$ & $11,6194^{\mathrm{A}}$ & $15,3840^{\mathrm{A}}$ & \\
\hline & 'Rubi MG-1192' & $6,3502^{\mathrm{B}}$ & $26,5939^{\mathrm{A}}$ & $10,0347^{\mathrm{A}}$ & $14,3263^{\mathrm{A}}$ & \\
\hline & ‘Catuaí Vermelho IAC-99’ & $11,7024^{\mathrm{AB}}$ & $16,2679^{\mathrm{A}}$ & $11,7480^{\mathrm{A}}$ & $13,2394^{\mathrm{A}}$ & \\
\hline & Média & $17,3723^{\text {a }}$ & $18,6140^{\mathrm{a}}$ & $11,1878^{\mathrm{a}}$ & & \\
\hline \multirow[t]{5}{*}{$\mathrm{Cu}$} & ‘Acaiá IAC-474-19' & $2,8441^{\mathrm{A}}$ & $2,0351^{\mathrm{A}}$ & $1,1537^{\mathrm{A}}$ & $2,0110^{\mathrm{A}}$ & \multirow{5}{*}{69,82} \\
\hline & 'Icatu Amarelo IAC-3282' & $1,9707^{\mathrm{A}}$ & $2,2686^{\mathrm{A}}$ & $1,2628^{A}$ & $1,8340^{\mathrm{A}}$ & \\
\hline & 'Rubi MG-1192' & $0,7502^{\mathrm{A}}$ & $3,2679^{A}$ & $0,9950^{\mathrm{A}}$ & $1,6710^{\mathrm{A}}$ & \\
\hline & ‘Catuaí Vermelho IAC-99' & $1,2758^{\mathrm{A}}$ & $2,1639^{A}$ & $1,0337^{\mathrm{A}}$ & $1,4911^{\mathrm{A}}$ & \\
\hline & Média & $1,7102^{\mathrm{a}}$ & $2,4339^{a}$ & $1,1113^{\mathrm{a}}$ & & \\
\hline \multirow[t]{5}{*}{$\mathrm{Zn}$} & ‘Acaiá IAC-474-19’' & $3,1705^{\mathrm{A}}$ & $1,8850^{\mathrm{A}}$ & $1,4809^{\mathrm{A}}$ & $2,1788^{\mathrm{A}}$ & \multirow{5}{*}{63,35} \\
\hline & 'Icatu Amarelo IAC-3282' & $2,3836^{\mathrm{A}}$ & $2,3675^{\mathrm{A}}$ & $1,4703^{\mathrm{A}}$ & $2,0738^{\mathrm{A}}$ & \\
\hline & 'Rubi MG-1192' & $1,1397^{\mathrm{A}}$ & $3,4170^{\mathrm{A}}$ & $1,1478^{\mathrm{A}}$ & $1,9015^{\mathrm{A}}$ & \\
\hline & 'Catuaí Vermelho IAC-99' & $1,1386^{\mathrm{A}}$ & $2,1275^{\mathrm{A}}$ & $1,5351^{\mathrm{A}}$ & $1,6004^{\mathrm{A}}$ & \\
\hline & Média & $1,9581^{\mathrm{a}}$ & $2,4492^{\mathrm{a}}$ & $1,4085^{\mathrm{a}}$ & & \\
\hline \multirow[t]{5}{*}{ B } & ‘Acaiá IAC-474-19’ & $2,6484^{\mathrm{A}}$ & $1,8678^{\mathrm{A}}$ & $1,2985^{\mathrm{A}}$ & $1,9382^{\mathrm{A}}$ & \\
\hline & 'Icatu Amarelo IAC-3282' & $1,6891^{\mathrm{A}}$ & $2,3218^{\mathrm{A}}$ & $1,3054^{\mathrm{A}}$ & $1,7721^{\mathrm{A}}$ & \\
\hline & 'Rubi MG-1192' & $0,4914^{\mathrm{A}}$ & $3,3068^{A}$ & $1,1985^{\mathrm{A}}$ & $1,6656^{\mathrm{A}}$ & 75,39 \\
\hline & 'Catuaí Vermelho IAC-99' & $0,9925^{\mathrm{A}}$ & $1,9445^{\mathrm{A}}$ & $1,2679^{\mathrm{A}}$ & $1,4016^{\mathrm{A}}$ & \\
\hline & Média & $1,4554^{\mathrm{a}}$ & $2,3602^{a}$ & $1,2676^{\mathrm{a}}$ & & \\
\hline
\end{tabular}

Médias seguidas pela mesma letra maiúscula na coluna e minúscula na linha não diferem estatisticamente entre si a $10 \%$, pelo teste de Tukey. 
Tabela 2 - Médias de eficiência de utilização de nutrientes para produção de caule, em $\mathrm{g}^{2} \mathrm{mg}^{-1} \mathrm{e} \mathrm{g}^{2} \mu \mathrm{g}^{-1}$ para macro e micronutrientes, respectivamente, de quatro cultivares de café arábica, tratadas com três níveis de adubação.

\begin{tabular}{|c|c|c|c|c|c|c|}
\hline \multirow{2}{*}{ Nutriente } & \multirow{2}{*}{ Cultivar } & \multicolumn{3}{|c|}{ 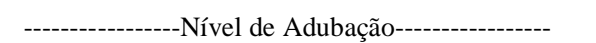 } & \multirow{2}{*}{ Média } & \multirow{2}{*}{$\mathrm{CV}(\%)$} \\
\hline & & Baixo & Normal & Alto & & \\
\hline \multirow[t]{5}{*}{$\mathrm{N}$} & 'Acaiá IAC-474-19' & $8,2345^{\mathrm{A}}$ & $6,6374^{\mathrm{A}}$ & $4,4864^{\mathrm{A}}$ & $6,4528^{\mathrm{A}}$ & \multirow{5}{*}{40,98} \\
\hline & 'Icatu Amarelo IAC-3282' & $3,7106^{\mathrm{B}}$ & $2,9328^{\text {в }}$ & $1,7717^{\mathrm{B}}$ & $2,8050^{\mathrm{A}}$ & \\
\hline & 'Rubi MG-1192' & $4,0075^{\mathrm{B}}$ & $1,9200^{\mathrm{B}}$ & $1,8417^{\mathrm{B}}$ & $2,5897^{\mathrm{A}}$ & \\
\hline & ‘Catuaí Vermelho IAC-99' & $1,5208^{\mathrm{C}}$ & $1,7466^{\mathrm{B}}$ & $1,4154^{\mathrm{B}}$ & $1,5609^{\mathrm{A}}$ & \\
\hline & Média & $4,3683^{\text {a }}$ & $3,3092^{b}$ & $2,3788^{b}$ & & \\
\hline \multirow[t]{5}{*}{$\mathrm{P}$} & ‘Acaiá IAC-474-19’ & $89,2536^{\mathrm{A}}$ & $106,8781^{\mathrm{A}}$ & $76,5792^{\mathrm{A}}$ & $90,9036^{\mathrm{A}}$ & \multirow{5}{*}{38,22} \\
\hline & 'Icatu Amarelo IAC-3282' & $47,5190^{\mathrm{B}}$ & $48,9235^{\text {в }}$ & $32,9632^{\mathrm{C}}$ & $43,1352^{\mathrm{A}}$ & \\
\hline & 'Rubi MG-1192' & $41,4689^{\mathrm{C}}$ & $32,0786^{\mathrm{C}}$ & $36,0371^{\text {в }}$ & $36,5282^{\mathrm{A}}$ & \\
\hline & ‘Catuaí Vermelho IAC-99' & $16,3877^{\mathrm{D}}$ & $27,8757^{\mathrm{D}}$ & $25,6289^{\mathrm{D}}$ & $23,2974^{\mathrm{A}}$ & \\
\hline & Média & $48,6573^{a}$ & $53,9389^{\mathrm{a}}$ & $42,8021^{\mathrm{a}}$ & & \\
\hline \multirow[t]{5}{*}{ S } & ‘Acaiá IAC-474-19’ & $109,3930^{\mathrm{A}}$ & $99,3523^{\mathrm{A}}$ & $62,3598^{A}$ & $90,3684^{\mathrm{A}}$ & \multirow{5}{*}{43,03} \\
\hline & 'Icatu Amarelo IAC-3282' & $49,0368^{\text {в }}$ & $40,9104^{\text {B }}$ & $25,2228^{\text {B }}$ & $38,3900^{\mathrm{A}}$ & \\
\hline & 'Rubi MG-1192' & $45,3472^{\mathrm{C}}$ & $27,4413^{\mathrm{C}}$ & $24,3533^{\text {в }}$ & $32,3806^{\mathrm{A}}$ & \\
\hline & ‘Catuaí Vermelho IAC-99’ & $18,6001^{\mathrm{D}}$ & $24,5467^{\mathrm{D}}$ & $19,5614^{\mathrm{C}}$ & $20,9027^{\mathrm{A}}$ & \\
\hline & Média & $55,5943^{\mathrm{a}}$ & $48,0627^{\text {a }}$ & $32,8743^{b}$ & & \\
\hline \multirow[t]{5}{*}{ K } & ‘Acaiá IAC-474-19’ & $16,8575^{\mathrm{A}}$ & $16,2754^{\mathrm{A}}$ & $10,0726^{\mathrm{A}}$ & $14,4018^{\mathrm{A}}$ & \multirow{5}{*}{47,89} \\
\hline & 'Icatu Amarelo IAC-3282' & $7,6674^{\mathrm{B}}$ & $6,4021^{\text {в }}$ & $3,8774^{\mathrm{B}}$ & $5,9823^{\mathrm{A}}$ & \\
\hline & 'Rubi MG-1192' & $8,3015^{\mathrm{B}}$ & $4,6594^{\mathrm{C}}$ & $4,3670^{\mathrm{B}}$ & $5,7760^{\mathrm{A}}$ & \\
\hline & ‘Catuaí Vermelho IAC-99' & $3,0178^{\mathrm{C}}$ & $3,8266^{\mathrm{C}}$ & $3,0158^{\mathrm{B}}$ & $3,2867^{\mathrm{A}}$ & \\
\hline & Média & $8,9610^{\text {a }}$ & $7,7909^{a b}$ & $5,3332^{b}$ & & \\
\hline \multirow[t]{5}{*}{$\mathrm{Ca}$} & ‘Acaiá IAC-474-19’ & $18,0379^{\mathrm{A}}$ & $18,0643^{\mathrm{A}}$ & $14,2529^{\mathrm{A}}$ & $16,7850^{\mathrm{A}}$ & \multirow{5}{*}{33,34} \\
\hline & 'Icatu Amarelo IAC-3282' & $8,1056^{\mathrm{B}}$ & $7,9441^{\text {в }}$ & $6,2656^{\mathrm{B}}$ & $7,4384^{\mathrm{A}}$ & \\
\hline & 'Rubi MG-1192' & $7,7137^{\mathrm{B}}$ & $5,7799^{\mathrm{C}}$ & $5,8702^{\text {в }}$ & $6,4546^{\mathrm{A}}$ & \\
\hline & 'Catuaí Vermelho IAC-99’ & $3,5374^{\mathrm{C}}$ & $4,2241^{\mathrm{C}}$ & $4,8539^{\mathrm{B}}$ & $4,2051^{\mathrm{A}}$ & \\
\hline & Média & $9,3486^{\mathrm{a}}$ & $9,0031^{\mathrm{a}}$ & $7,8106^{\mathrm{a}}$ & & \\
\hline \multirow[t]{5}{*}{$\mathrm{Mg}$} & 'Acaiá IAC-474-19’ & $56,8062^{\mathrm{A}}$ & $51,5025^{\mathrm{A}}$ & $37,0891^{\mathrm{A}}$ & $48,4659^{\mathrm{A}}$ & \multirow{5}{*}{34,82} \\
\hline & 'Icatu Amarelo IAC-3282' & $24,7607^{\text {B }}$ & $22,4005^{\text {B }}$ & $15,5802^{\text {В }}$ & $20,9138^{\text {A }}$ & \\
\hline & 'Rubi MG-1192' & $24,9659^{\text {в }}$ & $15,9332^{\mathrm{C}}$ & $16,3265^{\text {в }}$ & $19,0752^{\mathrm{A}}$ & \\
\hline & 'Catuaí Vermelho IAC-99' & $10,9470^{\mathrm{C}}$ & $12,4562^{\mathrm{D}}$ & $13,1347^{\mathrm{C}}$ & $12,1793^{\mathrm{A}}$ & \\
\hline & Média & $29,3699^{\text {a }}$ & $25,5731^{\mathrm{ab}}$ & $20,5326^{b}$ & & \\
\hline \multirow[t]{5}{*}{$\mathrm{Cu}$} & ‘Acaiá IAC-474-19’ & $7,0183^{\mathrm{A}}$ & $8,3318^{\mathrm{A}}$ & $4,4655^{\mathrm{A}}$ & $6,6052^{\mathrm{A}}$ & \multirow{5}{*}{36,82} \\
\hline & 'Icatu Amarelo IAC-3282' & $3,1455^{\mathrm{B}}$ & $3,5615^{\text {в }}$ & $1,7883^{\mathrm{B}}$ & $2,8318^{\mathrm{A}}$ & \\
\hline & 'Rubi MG-1192' & $2,8907^{\mathrm{BC}}$ & $2,2176^{\mathrm{B}}$ & $1,8230^{\mathrm{B}}$ & $2,3105^{\mathrm{A}}$ & \\
\hline & 'Catuaí Vermelho IAC-99' & $1,2875^{\mathrm{C}}$ & $1,9960^{\mathrm{B}}$ & $1,2380^{\mathrm{B}}$ & $1,5072^{\mathrm{A}}$ & \\
\hline & Média & $3,5855^{\mathrm{a}}$ & $4,0267^{\mathrm{a}}$ & $2,3287^{b}$ & & \\
\hline \multirow[t]{6}{*}{$\mathrm{Zn}$} & ‘Acaiá IAC-474-19’ & $14,0611^{\mathrm{A}}$ & $11,3487^{\mathrm{A}}$ & $7,9188^{\mathrm{A}}$ & $11,1096^{\mathrm{A}}$ & \multirow{4}{*}{42,10} \\
\hline & 'Icatu Amarelo IAC-3282' & $6,2716^{\mathrm{B}}$ & $4,9596^{\mathrm{B}}$ & $3,0698^{\mathrm{B}}$ & $4,7670^{\mathrm{A}}$ & \\
\hline & 'Rubi MG-1192' & $6,4053^{\text {в }}$ & $3,6656^{\mathrm{BC}}$ & $3,2574^{\mathrm{B}}$ & $4,4428^{\mathrm{A}}$ & \\
\hline & ‘Catuaí Vermelho IAC-99’ & $2,3801^{\mathrm{C}}$ & $3,1066^{\mathrm{C}}$ & $2,5399^{\mathrm{B}}$ & $2,6755^{\mathrm{A}}$ & \\
\hline & Média & $7,2795^{\mathrm{a}}$ & $5,7701^{a b}$ & $4,1965^{\mathrm{b}}$ & & \\
\hline & ‘Acaiá IAC-474-19’ & $4,8091^{\mathrm{A}}$ & $8,1057^{\mathrm{A}}$ & $4,2538^{A}$ & $5,7229^{\mathrm{A}}$ & \\
\hline \multirow{4}{*}{ B } & 'Icatu Amarelo IAC-3282' & $2,3064^{\mathrm{B}}$ & $3,7094^{\mathrm{B}}$ & $1,9503^{\mathrm{B}}$ & $2,6554^{\mathrm{A}}$ & \\
\hline & 'Rubi MG-1192' & $1,9432^{\mathrm{B}}$ & $2,1668^{\mathrm{BC}}$ & $2,1194^{\mathrm{B}}$ & $2,0765^{\mathrm{A}}$ & 48,38 \\
\hline & 'Catuaí Vermelho IAC-99' & $0,9199^{\mathrm{B}}$ & $1,7646^{\mathrm{C}}$ & $1,5837^{\text {B }}$ & $1,4228^{\mathrm{A}}$ & \\
\hline & Média & $2,4947^{b}$ & $3,9366^{\mathrm{a}}$ & $2,4768^{b}$ & & \\
\hline
\end{tabular}

Médias seguidas pela mesma letra maiúscula na coluna e minúscula na linha não diferem estatisticamente entre si a $10 \%$, pelo teste de Tukey. 
Tabela 3 - Médias de eficiência de utilização de nutrientes para produção de ramos, em $\mathrm{g}^{2} \mathrm{mg}^{-1} \mathrm{e} \mathrm{g}^{2} \mu \mathrm{g}^{-1}$ para macro e micronutrientes, respectivamente, de quatro cultivares de café arábica, tratadas com três níveis de adubação.

\begin{tabular}{|c|c|c|c|c|c|c|}
\hline \multirow{2}{*}{ Nutriente } & \multirow{2}{*}{ Cultivar } & \multicolumn{3}{|c|}{--------------Nível de Adubação-------------- } & \multirow{2}{*}{ Média } & \multirow{2}{*}{$\begin{array}{l}\mathrm{CV} \\
(\%)\end{array}$} \\
\hline & & Baixo & Normal & Alto & & \\
\hline \multirow[t]{5}{*}{$\mathrm{N}$} & 'Acaiá IAC-474-19' & $4,9600^{\mathrm{AB}}$ & $7,1425^{\mathrm{A}}$ & $6,7525^{\mathrm{A}}$ & $6,2850^{\mathrm{A}}$ & \multirow{5}{*}{35,94} \\
\hline & 'Icatu Amarelo IAC-3282' & $7,9175^{\mathrm{A}}$ & $7,3675^{\mathrm{A}}$ & $3,7650^{\mathrm{A}}$ & $6,3500^{\mathrm{A}}$ & \\
\hline & 'Rubi MG-1192' & $7,76000^{\mathrm{AB}}$ & $6,4075^{\mathrm{A}}$ & $4,8150^{\mathrm{A}}$ & $6,3275^{\mathrm{A}}$ & \\
\hline & ‘Catuaí Vermelho IAC-99' & $4,1000^{\mathrm{B}}$ & $6,8525^{\mathrm{A}}$ & $5,4500^{\mathrm{A}}$ & $5,4675^{\mathrm{A}}$ & \\
\hline & Média & $6,1844^{\mathrm{a}}$ & $6,9425^{\mathrm{a}}$ & $5,1956^{\mathrm{a}}$ & & \\
\hline \multirow[t]{5}{*}{$\mathrm{P}$} & 'Acaiá IAC-474-19' & $53,6225^{\mathrm{A}}$ & $114,9200^{\mathrm{A}}$ & $116,0900^{\mathrm{A}}$ & $94,8775^{\mathrm{A}}$ & \multirow{5}{*}{38,51} \\
\hline & 'Icatu Amarelo IAC-3282' & $101,5375^{\mathrm{A}}$ & $123,0125^{\mathrm{A}}$ & $70,1550^{\mathrm{A}}$ & $98,2350^{\mathrm{A}}$ & \\
\hline & 'Rubi MG-1192' & $80,3025^{\mathrm{A}}$ & $107,7750^{\mathrm{A}}$ & $93,6175^{\mathrm{A}}$ & $93,8983^{\mathrm{A}}$ & \\
\hline & 'Catuaí Vermelho IAC-99' & $46,1250^{\mathrm{A}}$ & $111,3725^{\mathrm{A}}$ & $103,0800^{\mathrm{A}}$ & $86,8592^{\mathrm{A}}$ & \\
\hline & Média & $70,3969^{a}$ & $114,2700^{\mathrm{a}}$ & $95,7356^{\mathrm{a}}$ & & \\
\hline \multirow[t]{5}{*}{$\mathrm{S}$} & ‘Acaiá IAC-474-19’' & $65,9750^{\mathrm{AB}}$ & $106,7225^{\mathrm{A}}$ & $94,2350^{\mathrm{A}}$ & $88,9775^{\mathrm{A}}$ & \multirow{5}{*}{35,98} \\
\hline & 'Icatu Amarelo IAC-3282' & $104,5900^{\mathrm{A}}$ & $102,9075^{\mathrm{A}}$ & $53,7825^{\mathrm{A}}$ & $87,0933^{\mathrm{A}}$ & \\
\hline & 'Rubi MG-1192' & $88,9125^{\mathrm{AB}}$ & $91,5050^{\mathrm{A}}$ & $64,0475^{\mathrm{A}}$ & $81,4883^{\mathrm{A}}$ & \\
\hline & 'Catuaí Vermelho IAC-99' & $50,9250^{\mathrm{B}}$ & $96,8275^{\mathrm{A}}$ & $74,5375^{\mathrm{A}}$ & $74,0967^{\mathrm{A}}$ & \\
\hline & Média & $77,6006^{\mathrm{a}}$ & $99,4906^{\mathrm{a}}$ & $71,6506^{\mathrm{a}}$ & & \\
\hline \multirow[t]{5}{*}{ K } & ‘Acaiá IAC-474-19’ & $10,1925^{\mathrm{A}}$ & $17,4100^{\mathrm{A}}$ & $15,4600^{\mathrm{A}}$ & $14,3542^{\mathrm{A}}$ & \multirow{5}{*}{39,37} \\
\hline & 'Icatu Amarelo IAC-3282' & $16,4950^{\mathrm{A}}$ & $16,0850^{\mathrm{A}}$ & $8,2400^{\mathrm{A}}$ & $13,6067^{\mathrm{A}}$ & \\
\hline & 'Rubi MG-1192' & $16,0700^{\mathrm{A}}$ & $15,6600^{\mathrm{A}}$ & $11,4300^{\mathrm{A}}$ & $14,3867^{\mathrm{A}}$ & \\
\hline & 'Catuaí Vermelho IAC-99' & $8,1950^{\mathrm{A}}$ & $15,0075^{\mathrm{A}}$ & $11,9525^{\mathrm{A}}$ & $11,7183^{\mathrm{A}}$ & \\
\hline & Média & $12,7381^{\mathrm{a}}$ & $16,0406^{\mathrm{a}}$ & $11,7706^{\mathrm{a}}$ & & \\
\hline \multirow[t]{5}{*}{$\mathrm{Ca}$} & ‘Acaiá IAC-474-19’ & $10,8475^{\mathrm{A}}$ & $19,4950^{\mathrm{A}}$ & $21,0050^{\mathrm{A}}$ & $17,1158^{\mathrm{A}}$ & \multirow{5}{*}{37,60} \\
\hline & 'Icatu Amarelo IAC-3282' & $17,4950^{\mathrm{A}}$ & $19,9600^{\mathrm{A}}$ & $13,4500^{\mathrm{A}}$ & $16,9683^{A}$ & \\
\hline & 'Rubi MG-1192' & $15,6300^{\mathrm{A}}$ & $19,2675^{\mathrm{A}}$ & $15,3450^{\mathrm{A}}$ & $16,7475^{\mathrm{A}}$ & \\
\hline & 'Catuaí Vermelho IAC-99' & $9,5550^{\mathrm{A}}$ & $16,7275^{\mathrm{A}}$ & $19,1550^{\mathrm{A}}$ & $15,1458^{\mathrm{A}}$ & \\
\hline & Média & $13,3819^{\mathrm{a}}$ & $18,8625^{\mathrm{a}}$ & $17,2388^{\mathrm{a}}$ & & \\
\hline \multirow[t]{5}{*}{$\mathrm{Mg}$} & ‘Acaiá IAC-474-19' & $34,0525^{\mathrm{A}}$ & $55,6325^{\mathrm{A}}$ & $55,0625^{\mathrm{A}}$ & $48,2492^{\mathrm{A}}$ & \multirow{5}{*}{33,59} \\
\hline & 'Icatu Amarelo IAC-3282' & $52,8825^{\mathrm{A}}$ & $56,2175^{\mathrm{A}}$ & $33,4225^{\mathrm{A}}$ & $47,5075^{\mathrm{A}}$ & \\
\hline & 'Rubi MG-1192' & $49,1550^{\mathrm{A}}$ & $53,1750^{\mathrm{A}}$ & $42,4500^{\mathrm{A}}$ & $48,2600^{\mathrm{A}}$ & \\
\hline & 'Catuaí Vermelho IAC-99' & $29,5800^{\mathrm{A}}$ & $49,7325^{A}$ & $48,3300^{\mathrm{A}}$ & $42,5475^{\mathrm{A}}$ & \\
\hline & Média & $41,4175^{\mathrm{a}}$ & $53,6894^{\mathrm{a}}$ & $44,8163^{\mathrm{a}}$ & & \\
\hline \multirow[t]{5}{*}{$\mathrm{Cu}$} & 'Acaiá IAC-474-19' & $4,2250^{\mathrm{A}}$ & $9,0450^{\mathrm{A}}$ & $6,7900^{\mathrm{A}}$ & $6,6867^{\mathrm{A}}$ & \multirow{5}{*}{33,33} \\
\hline & 'Icatu Amarelo IAC-3282' & $6,6950^{A}$ & $8,9525^{\mathrm{A}}$ & $3,8350^{\mathrm{A}}$ & $6,4942^{A}$ & \\
\hline & 'Rubi MG-1192' & $5,6625^{\mathrm{A}}$ & $7,4075^{\mathrm{A}}$ & $4,7475^{\mathrm{A}}$ & $5,9392^{\mathrm{A}}$ & \\
\hline & 'Catuaí Vermelho IAC-99' & $3,5675^{\mathrm{A}}$ & $7,7575^{\mathrm{A}}$ & $4,4775^{\mathrm{A}}$ & $5,2675^{\mathrm{A}}$ & \\
\hline & Média & $5,0375^{b}$ & $8,2906^{\mathrm{a}}$ & $4,9625^{b}$ & & \\
\hline \multirow[t]{5}{*}{$\mathrm{Zn}$} & ‘Acaiá IAC-474-19' & $8,4650^{\mathrm{AB}}$ & $12,1550^{\mathrm{A}}$ & $11,9425^{\mathrm{A}}$ & $10,8542^{\mathrm{A}}$ & \multirow{5}{*}{34,02} \\
\hline & 'Icatu Amarelo IAC-3282' & $13,4250^{\mathrm{A}}$ & $12,4125^{\mathrm{A}}$ & $6,6200^{\mathrm{A}}$ & $10,8192^{\mathrm{A}}$ & \\
\hline & 'Rubi MG-1192’' & $12,4800^{\mathrm{A}}$ & $12,4975^{\mathrm{A}}$ & $8,5500^{\mathrm{A}}$ & $11,1758^{\mathrm{A}}$ & \\
\hline & 'Catuaí Vermelho IAC-99' & $6,3800^{\mathrm{B}}$ & $12,0650^{\mathrm{A}}$ & $8,4975^{\mathrm{A}}$ & $8,9808^{\mathrm{A}}$ & \\
\hline & Média & $10,1875^{a}$ & $12,2825^{\mathrm{a}}$ & $8,9025^{\mathrm{a}}$ & & \\
\hline \multirow[t]{5}{*}{ B } & ‘Acaiá IAC-474-19’' & $2,8875^{\mathrm{A}}$ & $8,6400^{\mathrm{A}}$ & $6,4925^{\mathrm{A}}$ & $6,0067^{\mathrm{A}}$ & \\
\hline & 'Icatu Amarelo IAC-3282' & $5,0800^{\mathrm{A}}$ & $9,3275^{\mathrm{A}}$ & $4,1275^{\mathrm{A}}$ & $6,1783^{A}$ & \\
\hline & 'Rubi MG-1192' & $3,9475^{\mathrm{A}}$ & $7,0750^{\mathrm{A}}$ & $5,5250^{\mathrm{A}}$ & $5,5158^{\mathrm{A}}$ & 37,14 \\
\hline & 'Catuaí Vermelho IAC-99' & $2,5150^{\mathrm{A}}$ & $7,1350^{\mathrm{A}}$ & $6,0150^{\mathrm{A}}$ & $5,2217^{\mathrm{A}}$ & \\
\hline & Média & $3,6075^{\mathrm{c}}$ & $8,0444^{\mathrm{a}}$ & $5,5400^{\mathrm{b}}$ & & \\
\hline
\end{tabular}

Médias seguidas pela mesma letra maiúscula na coluna e minúscula na linha não diferem estatisticamente entre si a $10 \%$, pelo teste de Tukey. 
Tabela 4 - Médias de eficiência de utilização de nutrientes para produção de folhas, $\mathrm{em} \mathrm{g}^{2} \mathrm{mg}^{-1} \mathrm{e} \mathrm{g}^{2} \mu \mathrm{g}^{-1}$ para macro e micronutrientes, respectivamente, de quatro cultivares de café arábica, tratadas com três níveis de adubação.

\begin{tabular}{|c|c|c|c|c|c|c|}
\hline \multirow{2}{*}{ Nutriente } & \multirow{2}{*}{ Cultivar } & \multicolumn{3}{|c|}{ 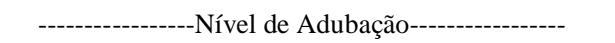 } & \multirow{2}{*}{ Média } & \multirow{2}{*}{$\mathrm{CV}(\%)$} \\
\hline & & Baixo & Normal & Alto & & \\
\hline \multirow[t]{5}{*}{$\mathrm{N}$} & 'Acaiá IAC-474-19' & $6,7494^{\mathrm{AB}}$ & $15,4400^{\mathrm{A}}$ & $11,6500^{\mathrm{A}}$ & $11,8275^{\mathrm{A}}$ & \multirow{5}{*}{44,08} \\
\hline & 'Icatu Amarelo IAC-3282' & $6,8300^{\mathrm{AB}}$ & $9,8525^{\mathrm{A}}$ & $8,0650^{\mathrm{A}}$ & $8,2492^{\mathrm{A}}$ & \\
\hline & 'Rubi MG-1192' & $14,0575^{\mathrm{A}}$ & $12,3625^{\mathrm{A}}$ & $9,2550^{\mathrm{A}}$ & $11,8917^{\mathrm{A}}$ & \\
\hline & 'Catuaí Vermelho IAC-99' & $4,5075^{\mathrm{B}}$ & $10,8575^{\mathrm{A}}$ & $6,4500^{\mathrm{A}}$ & $7,2717^{\mathrm{A}}$ & \\
\hline & Média & $8,4469^{a}$ & $12,1281^{\mathrm{a}}$ & $8,8550^{\mathrm{a}}$ & & \\
\hline \multirow[t]{5}{*}{$\mathrm{P}$} & ‘Acaiá IAC-474-19’ & $90,0525^{\mathrm{A}}$ & $248,9675^{\mathrm{A}}$ & $200,6675^{\mathrm{A}}$ & $179,8958^{\mathrm{A}}$ & \multirow{5}{*}{42,50} \\
\hline & 'Icatu Amarelo IAC-3282' & $87,2050^{\mathrm{A}}$ & $164,3150^{\mathrm{A}}$ & $150,8925^{\mathrm{A}}$ & $134,1375^{\mathrm{A}}$ & \\
\hline & 'Rubi MG-1192' & $145,9275^{\mathrm{A}}$ & $206,6800^{\mathrm{A}}$ & $182,1300^{\mathrm{A}}$ & $178,2458^{\mathrm{A}}$ & \\
\hline & 'Catuaí Vermelho IAC-99' & $53,6325^{\mathrm{A}}$ & $169,7875^{\mathrm{A}}$ & $116,6000^{\mathrm{A}}$ & $113,3400^{\mathrm{A}}$ & \\
\hline & Média & $94,2044^{\mathrm{b}}$ & $197,4375^{\text {a }}$ & $162,5725^{a}$ & & \\
\hline \multirow[t]{5}{*}{$\mathrm{S}$} & ‘Acaiá IAC-474-19’ & $110,7500^{\mathrm{A}}$ & $231,3800^{\mathrm{A}}$ & $162,7150^{\mathrm{A}}$ & $168,2817^{\mathrm{A}}$ & \multirow{5}{*}{45,24} \\
\hline & 'Icatu Amarelo IAC-3282' & $89,9525^{\mathrm{A}}$ & $137,4875^{\mathrm{A}}$ & $117,0075^{\mathrm{A}}$ & $114,8158^{\mathrm{AB}}$ & \\
\hline & 'Rubi MG-1192' & $161,6350^{\mathrm{A}}$ & $177,4250^{\mathrm{A}}$ & $121,8475^{\mathrm{A}}$ & $153,6358^{\mathrm{AB}}$ & \\
\hline & 'Catuaí Vermelho IAC-99' & $57,1425^{\mathrm{A}}$ & $152,3900^{\mathrm{A}}$ & $89,5300^{\mathrm{A}}$ & $99,6875^{\text {В }}$ & \\
\hline & Média & $104,8700^{b}$ & $174,6706^{\mathrm{a}}$ & $122,7750^{b}$ & & \\
\hline \multirow[t]{5}{*}{ K } & ‘Acaiá IAC-474-19’' & $17,1425^{\mathrm{AB}}$ & $37,8075^{\mathrm{A}}$ & $26,9100^{\mathrm{A}}$ & $27,2867^{\mathrm{A}}$ & \multirow{5}{*}{48,37} \\
\hline & 'Icatu Amarelo IAC-3282' & $14,0825^{\mathrm{AB}}$ & $21,5475^{\mathrm{A}}$ & $17,7750^{\mathrm{A}}$ & $17,8017^{\mathrm{A}}$ & \\
\hline & 'Rubi MG-1192' & $29,3425^{\mathrm{A}}$ & $30,1100^{\mathrm{A}}$ & $22,1425^{A}$ & $27,1983^{\mathrm{A}}$ & \\
\hline & 'Catuaí Vermelho IAC-99' & $9,1100^{\mathrm{B}}$ & $23,7875^{\mathrm{A}}$ & $13,7500^{\mathrm{A}}$ & $15,5492^{\mathrm{A}}$ & \\
\hline & Média & $17,4194^{\mathrm{a}}$ & $28,3131^{\text {a }}$ & $20,1444^{\mathrm{a}}$ & & \\
\hline \multirow[t]{5}{*}{$\mathrm{Ca}$} & ‘Acaiá IAC-474-19’' & $18,3025^{\mathrm{A}}$ & $42,2975^{\mathrm{A}}$ & $35,8425^{\mathrm{A}}$ & $32,1475^{A}$ & \multirow{5}{*}{40,82} \\
\hline & 'Icatu Amarelo IAC-3282' & $14,7900^{\mathrm{A}}$ & $26,6525^{\mathrm{A}}$ & $28,8250^{\mathrm{A}}$ & $23,4225^{\mathrm{A}}$ & \\
\hline & 'Rubi MG-1192' & $28,4375^{\mathrm{A}}$ & $37,6650^{\mathrm{A}}$ & $29,1825^{\mathrm{A}}$ & $31,7617^{\mathrm{A}}$ & \\
\hline & 'Catuaí Vermelho IAC-99' & $10,4075^{\mathrm{A}}$ & $25,6675^{A}$ & $22,0225^{\mathrm{A}}$ & $19,3658^{\mathrm{A}}$ & \\
\hline & Média & $17,9844^{\mathrm{b}}$ & $33,0706^{\mathrm{a}}$ & $28,9681^{\text {a }}$ & & \\
\hline \multirow[t]{5}{*}{$\mathrm{Mg}$} & ‘Acaiá IAC-474-19’' & $57,7675^{\mathrm{AB}}$ & $119,8150^{\mathrm{A}}$ & $94,2700^{\mathrm{A}}$ & $90,6175^{\mathrm{A}}$ & \multirow{5}{*}{39,68} \\
\hline & 'Icatu Amarelo IAC-3282' & $44,7950^{\mathrm{AB}}$ & $74,8350^{\mathrm{A}}$ & $71,3800^{\mathrm{A}}$ & $63,6700^{A}$ & \\
\hline & 'Rubi MG-1192' & $88,6775^{\mathrm{A}}$ & $103,0075^{\mathrm{A}}$ & $82,5650^{\mathrm{A}}$ & $91,4167^{\mathrm{A}}$ & \\
\hline & 'Catuaí Vermelho IAC-99' & $31,4575^{\text {В }}$ & $77,3550^{\mathrm{A}}$ & $59,1100^{\mathrm{A}}$ & $55,9742^{\mathrm{A}}$ & \\
\hline & Média & $55,6744^{\mathrm{b}}$ & $93,7531^{\text {a }}$ & $76,8313^{a b}$ & & \\
\hline \multirow[t]{5}{*}{$\mathrm{Cu}$} & ‘Acaiá IAC-474-19’ & $7,1350^{\mathrm{A}}$ & $19,4475^{\mathrm{A}}$ & $11,7700^{\mathrm{A}}$ & $12,7842^{\mathrm{A}}$ & \multirow{5}{*}{44,05} \\
\hline & 'Icatu Amarelo IAC-3282' & $5,7000^{\mathrm{A}}$ & $12,1300^{\mathrm{A}}$ & $8,3125^{\mathrm{A}}$ & $8,7142^{\mathrm{A}}$ & \\
\hline & 'Rubi MG-1192' & $10,3650^{\mathrm{A}}$ & $14,3975^{\mathrm{A}}$ & $9,1975^{\mathrm{A}}$ & $11,3200^{\mathrm{A}}$ & \\
\hline & 'Catuaí Vermelho IAC-99' & $4,1325^{\mathrm{A}}$ & $12,4475^{\mathrm{A}}$ & $5,7725^{\mathrm{A}}$ & $7,4508^{\mathrm{A}}$ & \\
\hline & Média & $6,8331^{\mathrm{b}}$ & $14,6056^{\mathrm{a}}$ & $8,7631^{\mathrm{b}}$ & & \\
\hline \multirow[t]{5}{*}{$\mathrm{Zn}$} & ‘Acaiá IAC-474-19’' & $14,3975^{\mathrm{AB}}$ & $26,4125^{\mathrm{A}}$ & $20,5625^{\mathrm{A}}$ & $20,4575^{\mathrm{A}}$ & \multirow{5}{*}{45,34} \\
\hline & 'Icatu Amarelo IAC-3282' & $11,4600^{\mathrm{AB}}$ & $16,4075^{\mathrm{A}}$ & $14,5025^{\mathrm{A}}$ & $14,1233^{\mathrm{A}}$ & \\
\hline & 'Rubi MG-1192' & $22,1625^{\mathrm{A}}$ & $23,4050^{\mathrm{A}}$ & $16,3275^{\mathrm{A}}$ & $20,6317^{\mathrm{A}}$ & \\
\hline & 'Catuaí Vermelho IAC-99' & $7,2700^{\mathrm{B}}$ & $19,3275^{\mathrm{A}}$ & $12,1650^{\mathrm{A}}$ & $12,9208^{A}$ & \\
\hline & Média & $13,8225^{\mathrm{a}}$ & $21,3881^{\mathrm{a}}$ & $15,8894^{\mathrm{a}}$ & & \\
\hline \multirow[t]{5}{*}{ B } & ‘Acaiá IAC-474-19' & $4,8075^{\mathrm{A}}$ & $18,5550^{\mathrm{A}}$ & $11,3175^{\mathrm{A}}$ & $11,5600^{\mathrm{A}}$ & \\
\hline & 'Icatu Amarelo IAC-3282' & $4,1800^{\mathrm{A}}$ & $12,3800^{\mathrm{AB}}$ & $8,5650^{\mathrm{A}}$ & $8,3750^{\mathrm{A}}$ & \\
\hline & 'Rubi MG-1192' & $7,1475^{\mathrm{A}}$ & $14,0775^{\mathrm{AB}}$ & $10,4150^{\mathrm{A}}$ & $10,5467^{\mathrm{A}}$ & 47,27 \\
\hline & 'Catuaí Vermelho IAC-99' & $2,8825^{\mathrm{A}}$ & $10,9975^{\mathrm{B}}$ & $6,8675^{\mathrm{A}}$ & $6,9158^{\mathrm{A}}$ & \\
\hline & Média & $4,7544^{\mathrm{c}}$ & $14,0025^{\mathrm{a}}$ & $9,2913^{b}$ & & \\
\hline
\end{tabular}

Médias seguidas pela mesma letra maiúscula na coluna e minúscula na linha não diferem estatisticamente entre si a $10 \%$, pelo teste de Tukey. 


\section{CONCLUSÃO}

A discriminação de cultivares de cafeeiro quanto à eficiência de utilização de nutrientes é mais evidente em solo com baixa disponibilidade destes. A cultivar 'Acaiá IAC-474-19' apresenta maior eficiência de utilização de macronutrientes para produção de raízes em solo com baixa disponibilidade de nutrientes e maior eficiência de utilização de nutrientes para produção de caule nos três níveis de adubação. A cultivar 'Icatu Amarelo IAC-3282' é a mais eficiente na utilização de $\mathrm{N}, \mathrm{S}$ e $\mathrm{Zn}$ para produção de ramos no nível baixo de adubação. A cultivar 'Rubi MG-1192' destaca-se quanto à eficiência de utilização de N, K, $\mathrm{Mg}$ e Zn para produção de folhas no nível baixo de adubação.

\section{AGRADECIMENTOS}

Ao Consórcio Nacional de Pesquisa e Desenvolvimento do Café (CNP\&D-Café), pelo financiamento do projeto.

\section{REFERÊNCIAS}

AMARAL, J.F.T. Eficiência de produção de raízes, absorção, translocação e utilização de nutrientes em cultivares de café arábica. 2002. 97f. Tese (Doutorado em Fitotecnia) - Universidade Federal de Viçosa, MG.

BALIGAR, V.C.; FAGERIA, N.K. Plant nutrient efficiency: towards the second paradigm. In: SIQUEIRA, J.O. et al. (Ed.). Inter-relação fertilidade, biologia do solo e nutrição de plantas. Lavras: Sociedade Brasileira de Ciência do Solo, 1998. p. $183-204$

CRUZ, C.D. Programa genes: aplicativo computacional em genética e estatística. Viçosa, MG: UFV, 2001. 648p.

DaMATTA, F.M. et al. Ecophysiology of coffee growth and production. Brazilian Journal of Plant Physiology, v.19, n.4, p.485-510, 2007. Disponível em: <http://www.scielo.br/ pdf/bjpp/v19n4/a14v19n4.pdf $>$. Acesso em: 21 abr. 2010. doi: 10.1590/S1677-04202007000400014.

DOBERMANN, A. et al. Management of phosphorus, potassium, and sulfur in intensive, irrigated lowland rice. Field Crops Research, v.56, p.113-138, 1998.

FAGERIA, N.K. et al. Rice cultivar evaluation for phosphorus use efficiency. Plant and Soil, n.111, p.105-109, 1988.

FAVARIN, J.L. et al. Equações para a estimativa do índice de área foliar do cafeeiro. Pesquisa Agropecuária Brasileira, v.37, n.6, p.769-773, 2002.

FÖHSE, D.; JUNGK, A. Influence of phosphate and nitrate supply on root hair formation of rape, spinach and tomato plants. Plant and Soil, n.74, p.359-368, 1983.

FURLANI, A.M.C. et al. Avaliação de genótipos de arroz quanto à eficiência na utilização de fósforo em solução nutritiva e em solo. Revista Brasileira de Ciência do Solo, v.7 n.7, p.291303, 1983.

GABELMAN, W.H.; GERLOFF, G.C. The search for and interpretation of genetic controls that enhance plant growth under deficiency levels of a macronutrient. Plant Soil, v.72, p.335-350, 1983.

GUIMARÃES, P.T.G. et al. (Ed.). Recomendações para o uso de corretivos e fertilizantes em Minas Gerais, $5^{\text {a }}$ Aproximação. Viçosa, MG: Comissão de Fertilidade do Solo do Estado de Minas Gerais - CFSEMG, 1999. p.289-302.

JONES JUNIOR, J.B. et al. Plant analysis handbook: a practical sampling, preparation, analysis, and interpretation guide. Athens, Georgia: Micro-Macro, 1991. $213 p$.

LAVIOLA, B.G. et al. Acúmulo de nutrientes em frutos de cafeeiro em quatro altitudes de cultivo: cálcio, magnésio e enxofre. Revista Brasileira de Ciência do Solo, v.31, n.6, p.1451-1462, 2007. Disponível em: <http://www.scielo.br/pdf/ rbcs/v31n6/22.pdf>. Acesso em: 21 abr. 2010. doi: 10.1590/ S0100-06832007000600022.

MALAVOLTA, E. Nutrição mineral e adubação do cafeeiro - colheitas econômicas máximas. São Paulo: Agronômica Ceres, 1993. 43p.

MANTOVANI, E.C.; COSTA, L.C. Manual do SISDA 2.0. In: WORKSHOP INTERNACIONAL SOBRE MANEJO INTEGRADO DAS CULTURAS E DOS RECURSOS HÍDRICOS, 1998, Brasília. Anais... Brasília, DF: SRH/UFV, 1998. 153p.

MARTINEZ, H.E.P. et al. Comportamento de variedades de soja cultivadas sob diferentes níveis de fósforo. II. Translocação do fósforo absorvido e eficiência nutricional. Revista Brasileira de Ciência do Solo, v.17, p.239-244, 1993.

PEREIRA, J.B.D. Eficiência nutricional de nitrogênio e de potássio em plantas de café (Coffea arabica L.). 1999. 99f. Tese (Doutorado em Fitotecnia) - Universidade Federal de Viçosa, MG.

SCHENK, M.K.; BARBER, S.A. Root characteristics of corn genotypes as related to P uptake. Agronomy Journal, n.71, p.921-924, 1979.

SIDDIQI, M.Y.; GLASS, A.D.M. Utilization index: a modified approach to the estimation and comparison of nutrient utilization efficiency in plants. Journal of Plant Nutrition, v.4, n.3, p.289-302, 1981.

SOUZA, R.B. Níveis críticos de enxofre em solos e folhas de cultivares de café. 1999. 88f. Tese (Doutorado em Fitotecnia) - Universidade Federal de Viçosa, MG.

TOMAZ, M.A.; AMARAL, J.F.T. Eficiência nutricional em plantas. Estudos avançados em produção vegetal. Alegre: Centro de Ciências Agrárias da Universidade Federal do Espírito Santo, 2008. V.1, p.23-41.

TOMAZ, M.A. et al. Eficiência de absorção, translocação e uso de cálcio, magnésio e enxofre por mudas enxertadas de Coffea arabica. Revista Brasileira de Ciência do Solo, v.27, n.5, p.885-892, 2003. Disponível em: <http:// www.scielo.br/pdf/rbcs/v27n5/v27n5a13.pdf>. Acesso em: 21 abr. 2010. doi: 10.1590/S0100-06832003000500013. 